\title{
Sliding Mode Force Control of an Electrohydraulic Servo System with RBF Neural Network Compensation
}

\author{
Xinliang LU*, Fengpo DU**, Qian JIA***, Bin REN****, Xingsong WANG***** \\ *School of Mechanical Engineering, Shijiazhuang Tiedao University, Shijiazhuang 050043, China, E-mail:xinref@126.com \\ **School of Mechanical Engineering, Southeast University, Nanjing 211189, China, E-mail: 230109036@seu.edu.cn \\ ***Industrial Center, Nanjing Institute of Technology, Nanjing 211167, China, E-mail: jiaqian@njit.edu.cn \\ ****School of Mechanical Engineering, Shijiazhuang Tiedao University, Shijiazhuang, China, E-mail:renbin@stdu.edu.cn \\ $* * * *$ School of Mechanical Engineering, Southeast University, Nanjing 211189, China, E-mail: xswang@seu.edu.cn \\ crossref http://dx.doi.org/10.5755/j01.mech.25.1.21279
}

\section{Nomenclature}

$x_{v}$ is spool servo valve displacement; $C_{d}$ is discharge coefficient; $W$ is spool servo valve area gradient; $\beta_{e}$ is effective bulk modulus; $K$ is spring stiffness coefficient; $f$ is external disturbance force; $Q_{1}, Q_{2}$ is cylinder chamber flow; $p_{s}$ is supply pressure; $p_{1}, p_{2}$ is pressure of chamber; $\rho$ is fluid density; $m$ is load mass; $B$ is viscous damping coefficient; $A_{1}, A_{2}$ is piston wording area; $C_{t}$ is total leakage coefficient; $u$ is control input signal; $x$ is displacement of piston; $V_{1}, V_{2}$ is cylinder chamber volume.

\section{Introduction}

The electrohydraulic servo system was widely used in the industrial field because it can provide large power [1]. But the hydraulic system shows highly inherent nonlinear characteristics which pose challenge to control design. The position control is relatively simple. But some industrial applications require force control, e.g. robots. However, the force control is more difficult than the position control for hydraulic system in the presence of uncertainties and strong nonlinearities.

This issue could be solved by the following two aspects. On the one hand, the hydraulic systems need be improved, including the development of advanced hydraulic servo valve. On the other hand, some advanced control algorithms were needed for force control of electrohydraulic systems. At this aspect, a lot of researchers have proposed some advanced algorithms. e.g. Wang proposed an output feedback domination approach for force control of an electrohydraulic actuator in the literature [2]. Alleyne and Zhu presented an adaptive approach to force control for electrohydraulic systems in the literature [3] and [4], respectively. Marusak presented a model predictive force control algorithms of an electrohydraulic actuator in the literature [5].

The sliding mode control is an effective method to design controller for nonlinear system. And it is robust to uncertainties [6]. In addition, the neural network technique is especially suitable for dealing with uncertain control issues [7]. To obtain accurate control, the neural network is employed to compensate the external disturbances because of its strong approximation ability for the unknown real functions [8]. Some researchers had combined the sliding mode control and neural network to resolve the issues of nonlinear control [9-11].

In this paper, the dynamics of hydraulic servo sys- tem is analyzed and a sliding mode controller with RBF neural network compensation is presented for the force tracking issue of electrohydraulic servo system. Because some state variables are needed in the controller, a state observer is designed to estimate the derivative and acceleration of force.

The remainder of this paper is organized as follows. The parameters meaning is presented in Section 1. The dynamics of electrohydraulic servo system is established in Section 2. The design of state observer is presented in Section 3. The sliding mode controller with RBF neural network compensation based on the Lyapunov method is addressed in Section 4. The experimental results of the force tracking performance are shown and analyzed in Section 5. The conclusion is drawn in Section 6.

\section{Dynamics of hydraulic servo system}

In this section, the dynamics of the hydraulic system is obtained that is a fundamental work of controller design. The hydraulic system that consists of a single-rod cylinder and an electro-hydraulic servo valve is shown in Fig. 1. The load is the combination of the inertia load and the elastic load.

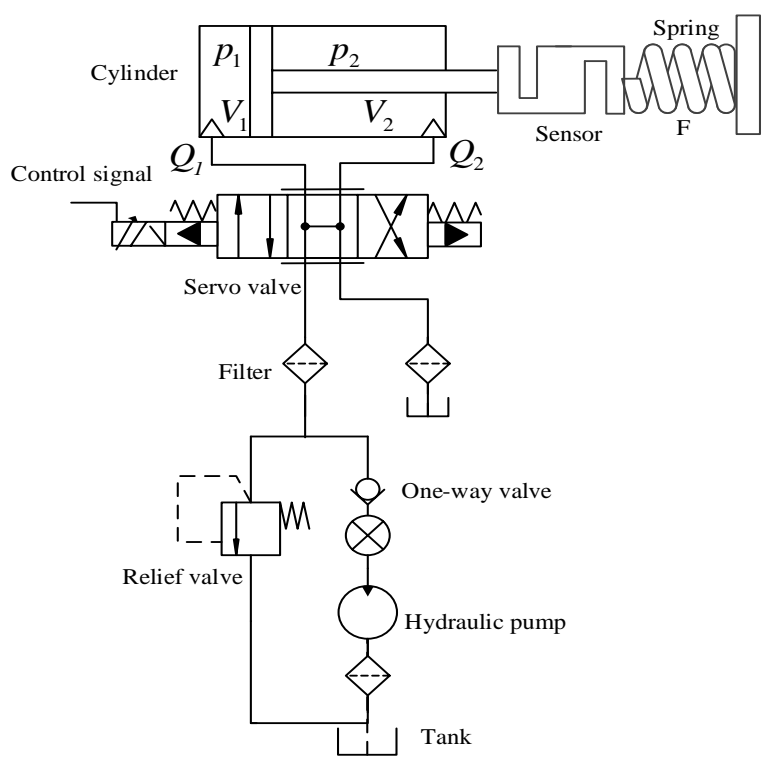

Fig. 1 Electro-hydraulic servo system follows.

$Q_{1}, Q_{2}$ is the function of $x_{v}$, they are presented as When $x_{v}>0$, servo valve flow equation is expressed 
as follows:

$$
\begin{aligned}
& Q_{1}=C_{d} W x_{v} \sqrt{2\left(p_{s}-p_{1}\right) / \rho} ; \\
& Q_{2}=C_{d} W x_{v} \sqrt{2 p_{2} / \rho} .
\end{aligned}
$$

When $x_{v}<0$, servo valve flow equation is expressed as follows:

$$
\begin{aligned}
& Q_{1}=C_{d} W x_{v} \sqrt{2 p_{1} / \rho} \\
& Q_{2}=C_{d} W x_{v} \sqrt{2\left(p_{s}-p_{2}\right) / \rho} .
\end{aligned}
$$

In order to reduce the difficulty of controller design, above equations must be simplified. We define the function as follows [12].

$$
s(*)=\left\{\begin{array}{l}
1, \text { if } * \geq 0 \\
0, \text { if } * \leq 0
\end{array}\right.
$$

Then the Eqs. (1-4) can be simplified and expressed uniformly as follows:

$$
\begin{aligned}
& Q_{1}=C_{d} W x_{v}\left[s\left(x_{v}\right) \sqrt{2\left(p_{s}-p_{1}\right) / \rho}+s\left(-x_{v}\right) \sqrt{2 p_{1} / \rho}\right] \\
& Q_{2}=C_{d} W x_{v}\left[s\left(x_{v}\right) \sqrt{2 p_{2} / \rho}+s\left(-x_{v}\right) \sqrt{2\left(p_{s}-p_{2}\right) / \rho}\right] .
\end{aligned}
$$

Where:

$$
x_{v}=\alpha u \text {. }
$$
trol input.

Where, $\alpha$ is the positive coefficient and $u$ is the con-

Then, Eqs. (6) and (7) is transformed to:

$$
\begin{aligned}
& Q_{1}=C_{d} \operatorname{Wau}\left[s\left(x_{v}\right) \sqrt{2\left(p_{s}-p_{1}\right) / \rho}+s\left(-x_{v}\right) \sqrt{2 p_{1} / \rho}\right] \\
& Q_{2}=C_{d} \operatorname{Wau}\left[s\left(x_{v}\right) \sqrt{2 p_{2} / \rho}+s\left(-x_{v}\right) \sqrt{2\left(p_{s}-p_{2}\right) / \rho}\right] .
\end{aligned}
$$

The cylinder flow continuity equation is given by [13]:

$$
\begin{aligned}
& A_{1} \dot{x}+C_{t}\left(p_{1}-p_{2}\right)+\frac{V_{1}+A_{1} x}{\beta_{e}} \dot{p}_{1}=Q_{1} ; \\
& A_{2} \dot{x}+C_{t}\left(p_{1}-p_{2}\right)=\frac{V_{2}-A_{2} x}{\beta_{e}} \dot{p}_{2}+Q_{2} .
\end{aligned}
$$

The dynamics of the load can be described by:

$$
A_{1} p_{1}-A_{2} p_{2}=m \frac{d^{2} x}{d t^{2}}+B \frac{d x}{d t}+k x+d
$$

Where, $d$ is the external disturbances and some unmodeled dynamics.

The force of the spring is $F=k x$, then $\dot{F}=k \dot{x}$ and
$\ddot{F}=k \ddot{x}$.

By combining Eq. (11-13), and $F, \dot{F}$ and are chosen as state variables, that implies the state variables is $x=\left[x_{1}, x_{2}, x_{3}\right]^{T} \triangleq[F, \dot{F}, \ddot{F}]^{T}$, then we can get the state equation as follows:

$$
\begin{aligned}
& \dot{x}_{1}=x_{2} \\
& \dot{x}_{2}=x_{3} \\
& \dot{x}_{3}=-\frac{A_{1} \beta_{e} C_{t}\left(p_{1}-p_{2}\right)}{m\left(V_{1}+\frac{A_{1}}{k} x_{1}\right)}-\frac{A_{2} \beta_{e} C_{t}\left(p_{1}-p_{2}\right)}{m\left(V_{2}-\frac{A_{2}}{k} x_{1}\right)}- \\
& -\left[\frac{A_{1}^{2} \beta_{e}}{m\left(k V_{1}+A_{1} x_{1}\right)}+\frac{A_{2}^{2} \beta_{e}}{m\left(k V_{2}-A_{2} x_{1}\right)}+\frac{1}{m}\right] x_{2}- \\
& -\frac{B}{k m} x_{3}+d(t)+ \\
& +\left[\frac{A_{1} \beta_{e} T_{1} k}{m\left(k V_{1}+A_{1} x_{1}\right)}+\frac{A_{2} \beta_{e} T_{2} k}{m\left(k V_{2}-A_{2} x_{1}\right)}\right] u .
\end{aligned}
$$

Where:

$$
\begin{aligned}
& T_{1}=C_{d} W \alpha\left[s\left(x_{v}\right) \sqrt{2\left(p_{s}-p_{1}\right) / \rho}+s\left(-x_{v}\right) \sqrt{2 p_{1} / \rho}\right], \\
& T_{2}=C_{d} W \alpha\left[s\left(x_{v}\right) \sqrt{2 p_{2} / \rho}+s\left(-x_{v}\right) \sqrt{2\left(p_{s}-p_{2}\right) / \rho}\right] .
\end{aligned}
$$

The simplified state equation is expressed as follows:

$$
\begin{aligned}
& \dot{x}_{1}=x_{2} \\
& \dot{x}_{2}=x_{3} \\
& \dot{x}_{3}=f\left(x_{1}, x_{2}\right)+\phi_{1} x_{3}+g\left(x_{1}, x_{2}\right) u+d(t) .
\end{aligned}
$$

Where:

$$
\begin{aligned}
& f\left(x_{1}, x_{2}\right)=-\frac{A_{1} \beta_{e} C_{t}\left(p_{1}-p_{2}\right)}{m\left(V_{1}+\frac{A_{1}}{k} x_{1}\right)}-\frac{A_{2} \beta_{e} C_{t}\left(p_{1}-p_{2}\right)}{m\left(V_{2}-\frac{A_{2}}{k} x_{1}\right)}- \\
& -\left[\frac{A_{1}^{2} \beta_{e}}{m\left(k V_{1}+A_{1} x_{1}\right)}+\frac{A_{2}^{2} \beta_{e}}{m\left(k V_{2}-A_{2} x_{1}\right)}+\frac{1}{m}\right] x_{2} ; \\
& \phi_{1}=-\frac{B}{k m} ; \\
& g(x)=\frac{A_{1} \beta_{e} T_{1} k}{m\left(k V_{1}+A_{1} x_{1}\right)}+\frac{A_{2} \beta_{e} T_{2} k}{m\left(k V_{2}-A_{2} x_{1}\right)} .
\end{aligned}
$$

\section{The design of state observer}

The value of the force $F$ can be obtained directly via the s-type force sensor. But $F$ cannot be directly obtained by differentiating the force signal because of the large amount of noises. In order to acquire relatively accurate values for the $\dot{F}$ and $\ddot{F}$, we design an observer to evaluate the values. The observer is defined as follows [14]: 


$$
\left\{\begin{array}{l}
\dot{\hat{x}}_{1}=\hat{x}_{2}-k_{1} \operatorname{sgn}\left(\hat{x}_{1}-x_{1}\right) \\
\dot{\hat{x}}_{2}=\hat{x}_{3}-k_{2} \operatorname{sgn}\left(\hat{x}_{1}-x_{1}\right) \\
\dot{\hat{x}}_{3}=f\left(\hat{x}_{1}, \hat{x}_{2}\right)+\phi_{1} \hat{x}_{3}+g\left(\hat{x}_{1}, \hat{x}_{2}\right) u-k_{3} \operatorname{sgn}\left(\hat{x}_{1}-x_{1}\right)
\end{array} .\right.
$$
spectively.

Where: $\hat{x}_{1}, \hat{x}_{2}, \hat{x}_{3}$ are the estimates of $x_{1}, x_{2}, x_{3}$ reten as follows:

$$
\left\{\begin{array}{l}
\dot{\tilde{x}}_{1}=\tilde{x}_{2}-k_{1} \operatorname{sgn}\left(\hat{x}_{1}-x_{1}\right) \\
\dot{\tilde{x}}_{2}=\tilde{x}_{3}-k_{2} \operatorname{sgn}\left(\hat{x}_{1}-x_{1}\right) \\
\dot{\tilde{x}}_{3}=\tilde{f}+\phi_{1} \tilde{x}_{3}+\tilde{g} u-d(t)-k_{3} \operatorname{sgn}\left(\hat{x}_{1}-x_{1}\right)
\end{array} .\right.
$$

Where:

$\tilde{f}=f\left(\hat{x}_{1}, \hat{x}_{2}\right)-f\left(x_{1}, x_{2}\right) ; \quad \tilde{g}=g\left(\hat{x}_{1}, \hat{x}_{2}\right)-g\left(x_{1}, x_{2}\right)$.

\section{Control design}

\subsection{Sliding mode controller}

A sliding mode force controller with RBF neural network compensation is presented in this section. As shown in Fig. 2 The controller is composed of a sliding mode controller as the primary controller and a RBF neural network controller which is employed to compensate the errors of model uncertainties and external disturbances.

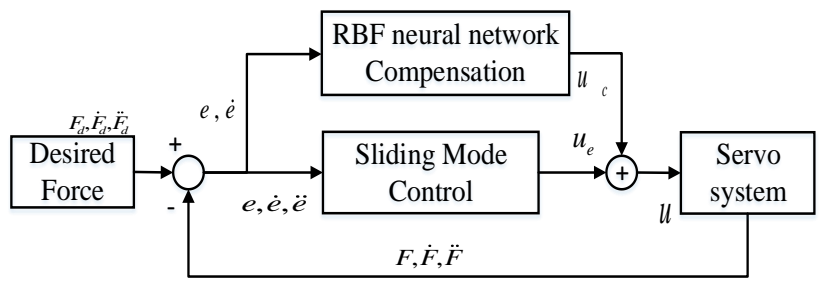

Fig. 2 Schematic of control architecture

Define force tracking error is:

$$
e=x_{1}-x_{d}
$$

Where $x_{d}$ is the desired force.

The sliding hyperplane is defined as follows [15]:

$$
s=c_{1} e+c_{2} \dot{e}+\ddot{e} .
$$

Where: $c_{1}>0, c_{2}>0$ and $c_{1}, c_{2}$ satisfy the Hurwitz stability requirements[16].

Differentiating the Eq. (19) and combining the Eq. (15), then following equation is obtained:

$$
\begin{aligned}
& \dot{s}=c_{1} \dot{e}+c_{2} \ddot{e}+\dddot{x}_{1}-\dddot{x}_{d}= \\
& =c_{1}\left(\dot{x}_{1}-\dot{x}_{d}\right)+c_{2}\left(\ddot{x}_{1}-\ddot{x}_{d}\right)+\dddot{x}_{1}-\dddot{x}_{d}= \\
& =c_{1} \hat{x}_{2}+c_{2} \hat{x}_{3}-\dddot{x}_{d}+f\left(\hat{x}_{1}, \hat{x}_{2}\right)+ \\
& +g\left(\hat{x}_{1}, \hat{x}_{2}\right) u+\phi_{1} \hat{x}_{3}+c_{1} \dot{x}_{d}+c_{2} \ddot{x}_{d}+d(t) .
\end{aligned}
$$

The candidate Laypunov function is defined as:

$$
V=\frac{1}{2} s^{2}
$$

When the control input is defined as follows:

$$
\begin{aligned}
& u=-\frac{1}{g\left(\hat{x}_{1}, \hat{x}_{2}\right)}\left(c_{1} \hat{x}_{2}+c_{2} \hat{x}_{3}+f\left(\hat{x}_{1}, \hat{x}_{2}\right)+\phi_{1} \hat{x}_{3}+\right. \\
& \left.+d(t)+c_{1} \dot{x}_{d}+c_{2} \ddot{x}_{d}-\dddot{x}_{d}+K_{d} s+K s g n(\mathrm{~s})\right) .
\end{aligned}
$$
is obtained:

According to Eqs. (20-22), the following inequality

$$
\dot{V}=s \dot{s}=-K_{d} s^{2}-K|s|<0 .
$$

\subsection{RBF neural network compensation}

The $d(t)$ is uncertain in Eq. (22), we can't get the accurate value in control input. The RBF neural network is used to get the estimate value of $d(t)$ due to the excellent approximation performance.

The $j_{t h}$ neuron output in RBF neural network is [17]:

$$
h_{j}=\exp \left(-\frac{\left\|x-c_{j}\right\|^{2}}{2 b_{j}^{2}}\right) .
$$

Where: $x=[F, \dot{F}]^{T}$ is the neural network input, $c_{j}$ is the central vector and $b_{j}$ is the base width vector.

The network weight vector is:

$$
\theta=\left[\theta_{1}, \cdots, \theta_{m}\right]^{T}
$$

Where: $m$ is the number of neural network nodes. RBF network output is:

$$
y_{m}(x)=\theta^{\mathrm{T}} h(x)
$$

For any given real continuous function $f(x)$, on a compact set $\Omega \subseteq R^{n}$ there exists a RBF network in the form of (26) such that $\forall \varepsilon>0[18]$.

$$
\sup _{x \in \Omega}\left|y_{m}(t)-f(x)\right|<\varepsilon
$$

The approximation errors are expressed as follows:

$$
e_{f}=|d(x)-\hat{d}(x)|
$$

The performance of RBF is set:

$$
E(k)=\frac{1}{2}\left(y(k)-y_{m}(k)\right)^{2} .
$$

The gradient descent method is adopted to adjust 
the weights of the network.

$$
\Delta \theta_{j}(k)=-\eta \frac{\partial E(k)}{\partial \theta_{j}(k)} .
$$

Where: $\eta$ is the learning factor.

The network weight adjustment is as follows:

$$
\theta(k)=\theta(k-1)+\Delta \theta(k)+\alpha(\theta(k-1)-\theta(k-2)) .
$$

Where: $\alpha$ is the momentum factor and $k$ is the iterative step.

When the control input is revised as follows:

$$
\begin{aligned}
& u=-\frac{1}{g\left(\hat{x}_{1}, \hat{x}_{2}\right)}\left(c_{1} \hat{x}_{2}+c_{2} \hat{x}_{3}+f\left(\hat{x}_{1}, \hat{x}_{2}\right)+\phi_{1} \hat{x}_{3}+\right. \\
& \left.+\theta^{\mathrm{T}} h(x)+c_{1} \dot{x}_{d}+c_{2} \ddot{x}_{d}-\dddot{x}_{d}+K_{d} s+K s g h(\mathrm{~s})\right) .
\end{aligned}
$$

Where: $K_{d}>0$ and $K>e_{f}$.

According to Eqs. (20-21) and (32), the following inequality is obtained:

$$
\dot{V}=s \dot{s}=-K_{d} s^{2}-\left|\left(K-e_{f}\right) s\right|<0 .
$$
cally stable.

This implies that the system is globally asymptoti-

\section{Experiment}

\subsection{Experimental setup}

As shown in Fig.3, the experimental platform is composed of a cylinder, a servo valve, a s-type force sensor, a spring, a hydraulic working station and two industrial control computers. The load is elastic load which is measured by the force sensor. In order to ensure the control algorithms are implemented in real-time, they are run in Matlab/Simulink Real-time Workshop environment.

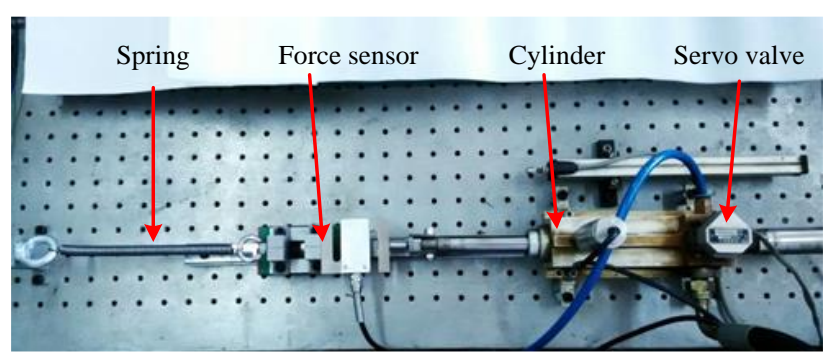

Fig. 3 Experimental platform

Two industrial personal computers communicate via the Ethernet, where one is the host computer which is used to compile the control algorithm easily and the other one is the target computer which is utilized to execute the control algorithm in real-time. A PCL-726 module which is installed in the target computer is employed to output control voltage between $-5 \mathrm{~V}-5 \mathrm{~V}$. The load force is directly measured by the sensor and acquired by a PCL-812PG module which is manufactured by Advantech Corporation.
5.2. parameters values

Table 2

The parameters of hydraulic servo system

\begin{tabular}{|c|c|}
\hline Symbols & Value \\
\hline$p_{s}$ & $5 \mathrm{Mpa}$ \\
\hline$A_{l}$ & $4.9 \times 10^{-4} \mathrm{~m}^{2}$ \\
\hline$A_{2}$ & $2.9 \times 10^{-4} \mathrm{~m}^{2}$ \\
\hline$m$ & $4.19 \mathrm{Kg}$ \\
\hline$A_{l}=A_{2}$ & $4.5 \times 10^{-5} \mathrm{~m}^{3}$ \\
\hline$\beta_{e}$ & $800 \mathrm{Mpa}$ \\
\hline$\rho$ & $850 \mathrm{~kg} / \mathrm{m}^{3}$ \\
\hline$C_{t}$ & $5 \times 10^{-13} \mathrm{~m}^{3} / \mathrm{Pa}$ \\
\hline$W$ & $1.2 \times 10^{-2}$ \\
\hline$C_{d}$ & 0.8 \\
\hline$k$ & $500 \mathrm{~N} / \mathrm{m}$ \\
\hline
\end{tabular}

\subsection{Experiment results}

To validate the effectiveness of the proposed sliding mode force control with BRF neural network compensation approach, the comparison of control performance of force tracking between the proposed control method and PID control is shown in Figs.4 to 9. Especially, the tracking performance experiments with the proposed algorithms verify its superiority to PID control when the disturbances are uncertain.

In the force tracking experiments of electrohydraulic system, the desired trajectory is set as a sine function $y=80+32 \sin (\pi t / 3)$. As shown in Fig. 4, the good tracking results can be obtained with well-tuned PID and the sliding mode control with RBF compensation algorithm without external disturbances. Fig. 5 shows the force tracking errors. However, the sliding mode control with RBF compensation algorithm can obtain better tracking performance and relatively small errors occur compared with PID under uncertain disturbances as shown in Figs. 6 and 7 shows the force tracking errors of the proposed algorithms and PID control. As shown in the Figs.6 and 7, the PID is most sensitive to the disturbances, but the proposed algorithm is robust to uncertain disturbances.

A sine function with variable frequency and amplitude was also chosen as the reference trajectory to further validate the performance of the proposed algorithms. As shown in Fig. 8, the reference trajectories are $y=80+32 \sin (\pi t / 3)$ before $12 \mathrm{~s}$ and $y=80+48 \sin (\pi t / 4)$ at the rest time, respectively.

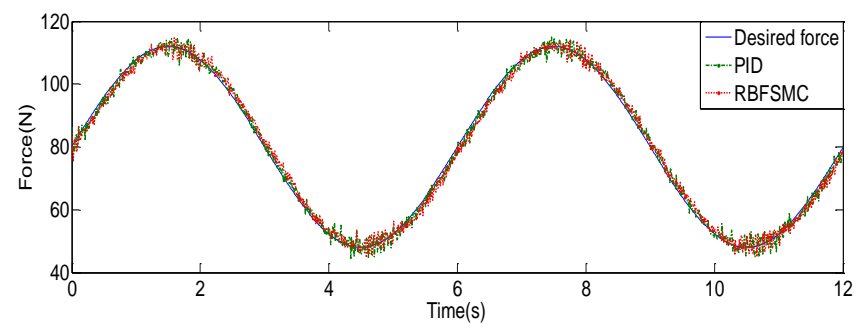

Fig. 4 The force tracking without external disturbances

Even the change of the frequency and amplitude, the proposed algorithm can always get good tracking performance. The errors of PID algorithm are larger than the errors of the proposed algorithm. 


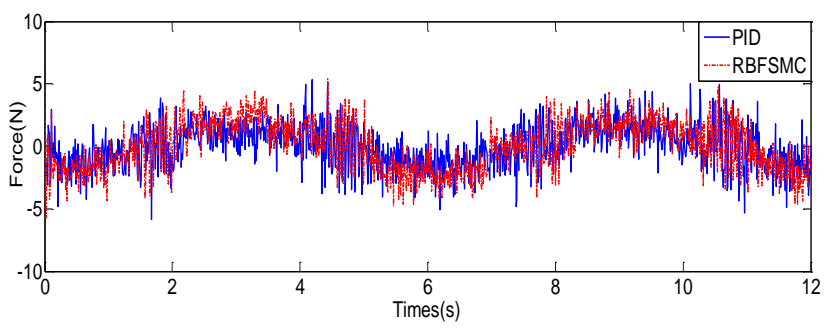

Fig. 5 The force tracking errors without external disturbances

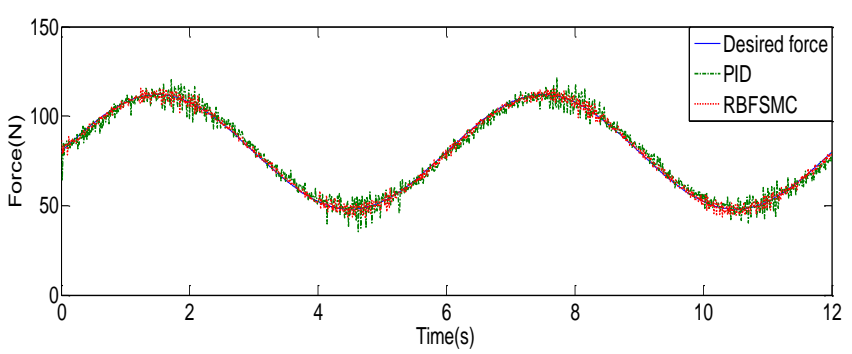

Fig. 6 The force tracking with uncertain disturbances

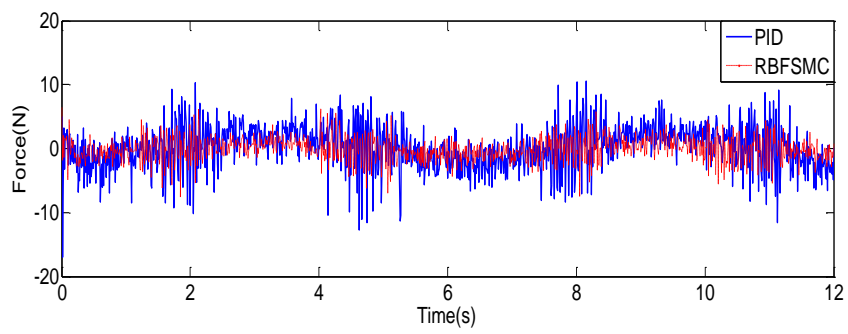

Fig. 7 The force tracking errors with uncertain disturbances

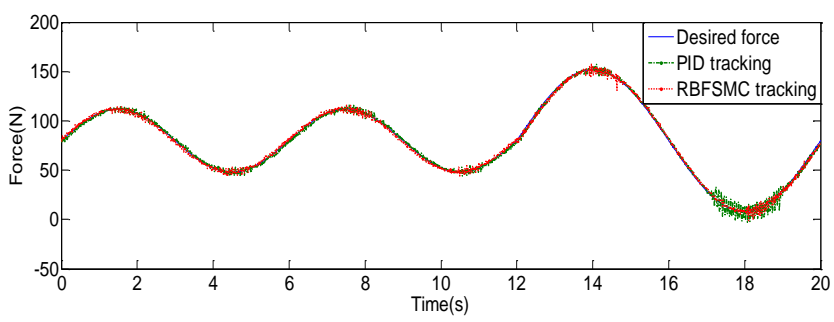

Fig. 8 The force tracking at the variable frequency and amplitude

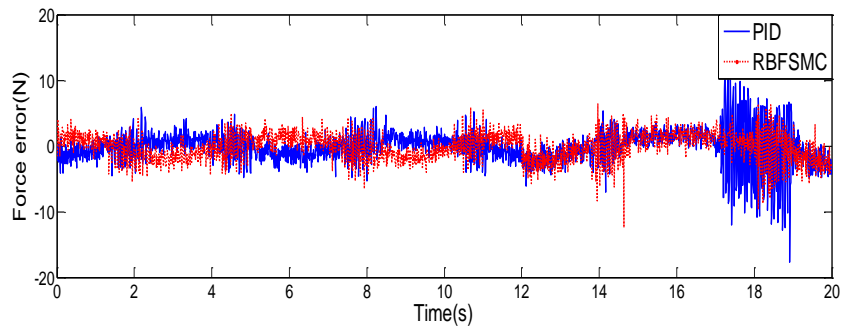

Fig. 9 The force tracking errors at the variable frequency and amplitude

\section{Conclusion}

In this paper, the dynamics of an electrohydraulic servo system is analyzed. Then, a sliding mode control approach with RBF neural network compensation was addressed for the force tracking control. The algorithm is globally asymptotically stable under the Lyapunov meaning. The experiments demonstrate the algorithms are effective and have better performances than the PID control. The algorithms also show the robustness in the presence of uncertainties and strong nonlinearities.

\section{Acknowledgements}

This work is supported by National Nature Science Foundation of China (Grant No. 51575100), Nature Science Foundation of Jiangsu Province (Grant No. BK20170766), High-level Talent Foundation of Hebei Province (Grant No. B2016003015), Top Talent Program Foundation in University of Hebei Province (Grant No. BJ2017047).

\section{References}

1. Guan, C.; Pan, S. 2008. Adaptive sliding mode control of electro-hydraulic system with nonlinear unknown parameters. Control Engineering Practice 16(11):12751284 . http://dx.doi.org/10.1016/j.conengprac.2008.02.002.

2. Wang, X.; Sun, X.; Li, S.; Ye, H. 2012. Output feedback domination approach for finite-time force control of an electrohydraulic actuator. IET Control Theory and Applications 6(7): 921-934.

http://dx.doi.org/10.1049/iet-cta.2011.0461/

3. Alleyne, A.; Liu, R. 2000. A simplified approach to force control for electro-hydraulic systems. Control Engineering Practice 8(12):1347-1356. http://dx.doi.org/10.1016/S0967-0661(00)00081-2.

4. Zhu, W.; Piedboeuf, J. 2004. Adaptive output force tracking control of hydraulic cylinders with applications to robot manipulators, Journal of Dynamic Systems Measurement, and Control 127(2): 206-217. http://dx.doi.org/10.1115/1.1898237.

5. Marusak, P.; Kuntanapreeda, S. 2011. Constrained model predictive force control of an electrohydraulic actuator. Control Engineering Practice 19(1): 62-73. http://dx.doi.org/10.1016/j.conengprac.2010.09.002.

6. Utkin, V.; Guldner, J.; Shi, J. 2009. Sliding mode control in electro-mechanical systems: Taylor \& Francis Group.

7. Yu, L.; Fei, S.; Sun, L.; Huang, J. 2013. An adaptive neural network switching control approach of robotic manipulators for trajectory tracking, International Journal of Computer Mathematics 91(5): 983-995. http://dx.doi.org/10.1080/00207160.2013.813021.

8. Liu, J. 2014. RBF neural network control for mechanical systems design, analysis and MATLAB simulation Beijing: Tsinghua University Press.

9. Mahjoub, S.; Mnif ,F.; Derbel, N.; Hamerlain, M. 2014. Radial-basis-functions neural network sliding mode control for underactuated mechanical systems, International journal of dynamics and control 2(4): 533541. http://dx.doi.org/10.1007/s40435-014-0070-0,

10. Zou, Y.; Lei, X. 2015. A compound control method based on the adaptive neural network and sliding mode control for inertial stable platform, Neurocomputing, 2015, 155(1):286-294.

http://dx.doi.org/10.1016/j.neucom.2014.12.074.

11. Mefoued, S. 2015. A second order sliding mode control and a neural network to drive a knee joint actuated orthosis, Neurocomputing 2015, 155 (1):71-79. 
http://dx.doi.org/10.1016/j.neucom.2014.12.047.

12. Tafazoli, S.; de Silva C.; Lawrence, P. 1998. Tracking control of an electrohydraulic manipulator in the presence of friction, IEEE Transactions on Control Systems Technology 1998, 6(3): 401-411. http://dx.doi.org/10.1109/87.668040,

13. Merritt, H. Hydraulic control systems. New York: Wiley. 1967

14. Chen, Y. 2010. Backstepping controller design for electro-hydraulic servo system with sliding observer, 29th Chinese Control Conference 2010:391-394.

15. Yang, Y.; Cheng, C. 2013. Robust adaptive trajectory control for an omnidirectional vehicle with parametric uncertainty, Transactions of the canadian society for mechanical engineering 37(3): 405-413.

16. Richard, C.; Bishop, R. 2011. Modern control systems (12th ed.), New Jersey: Prentice Hall.

17. Alam, J.; Hassan, M.; Khan, A.; Chaudhry, A. 2015. Robust fuzzy RBF network based image segmentation and intelligent decision making system for carotid artery ultrasound images, Neurocomputing 151(2): 745-755. http://dx.doi.org/10.1016/j.neucom.2014.10.027.

18. Huanqing, W.; Xiaoping, L.; Kefu L. 2015. Adaptive neural data-based compensation control of non-linear systems with dynamic uncertainties and input saturation. IET Control Theory \& Applications 9(7):1058-1065. http://dx.doi.org/10.1049/iet-cta.2014.0709.
Xinliang Lu, Fengpo Du, Qian Jia, Bin Ren, Xinsong Wang

\section{SLIDING MODE FORCE CONTROL OF AN ELEC- TROHYDRAULIC SERVO SYSTEM WITH RBF NEURAL NETWORK COMPENSATION}

\section{S u m m a ry}

In this paper, the dynamics of an electrohydraulic servo system is analyzed. It is difficult to achieve the precise force tracking control due to its high nonlinearities and parameter uncertainties. For the accurate force tracking control, a sliding mode control algorithm with radial basis function (RBF) neural network compensation was proposed. The theory verifies that the algorithm is globally asymptotically stable. The experimental results show that the proposed algorithm is not only effective and better than the PID control in the force tracking control, but also robust to external uncertain disturbances.

Key words: electrohydraulic, RBF, neural network, sliding mode, compensation control, trajectory tracking.

Received July 19, 2018

Accepted February 15, 2019 